\title{
Electroweak Radiative Corrections at High Energies
}

\author{
Ansgar Denner* \\ Paul Scherrer Institut, 5232 Villigen PSI, Switzerland \\ E-mail: 'Ansgar. Denner@psi.ch'
}

ABSTRACT: For energies far above the electroweak scale, large electroweak radiative corrections occur that grow logarithmically with energy and can easily reach several tens of per cent in the $\mathrm{TeV}$ range. Recent work on these corrections is reviewed.

\section{Introduction}

In the energy range above the electroweak scale, $\sqrt{s} \gg M_{\mathrm{W}}$, large electroweak radiative corrections occur, which are due to $\operatorname{logarithms} \log \left(s / M_{\mathrm{W}}^{2}\right)$ involving the ratio of the energy

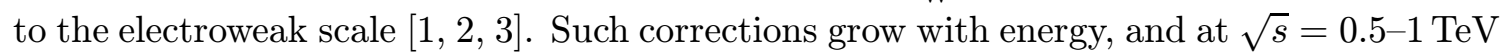
they are typically of order $10 \%$ of the theoretical prediction.

For electroweak processes that are not mass-suppressed at high energies, these logarithmic corrections are universal. On the one hand, single logarithms originating from short-distance scales are related to the renormalization of dimensionless parameters, i.e. the running of the gauge, Yukawa, and scalar couplings. These are governed by the renormalization group. On the other hand, based on our experience from QCD, universal logarithms originating from the long-distance scale $M_{\mathrm{W}} \ll \sqrt{s}$ are expected to factorize, i.e. they can be associated with external lines in Feynman diagrams. At the one-loop level, they consist of double-logarithmic and single-logarithmic terms originating from soft-collinear and collinear (or soft) gauge bosons, respectively, coupled to external legs. The non-logarithmic terms, on the other hand, are in general non-universal and have to be evaluated for each process separately, if needed.

In the recent literature most interest has been devoted to electroweak long-distance corrections. The main difference between QCD and the Electroweak Standard Model is that the masses of the weak gauge bosons provide a physical cut-off for real Z- and Wboson emission. Therefore, for a sufficiently good experimental resolution, soft and collinear weak-boson radiation need not be included in the theoretical predictions and, except for electromagnetic real corrections, one can restrict oneself to large logarithms originating from virtual corrections. This is assumed in Sects. 12 i 15 , if not stated otherwise.

${ }^{*}$ This work was supported in part by the Swiss Bundesamt für Bildung und Wissenschaft and by the European Union under contract HPRN-CT-2000-00149. 


\section{Explicit one-loop calculations}

One approach to the logarithmic electroweak corrections is via explicit calculation from Feynman diagrams. This approach has been mainly applied at the one-loop level and often starts from complete calculations of the one-loop corrections. As compared to the complete results, the results in logarithmic approximation are shorter and allow analytical studies of the structure of the corrections.

The first evaluation of electroweak corrections at high energies was already performed many years ago by Beenakker et al. [2: In this work, the electroweak corrections to on-shell W-pair production were evaluated in the high-energy limit including besides the logarithmic contributions also the constant terms.

More recently, the virtual logarithmic corrections to $\mathrm{e}^{+} \mathrm{e}^{-} \rightarrow f \bar{f}$ have been considered in a series of papers using explicit high-energy expansions for Feynman diagrams. Ciafaloni and Comelli [i] have pointed out the role of the Sudakov double logarithms and discussed their origin. Beccaria et al. have considered the complete logarithmic corrections and stud-

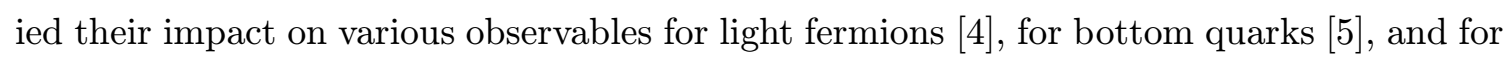

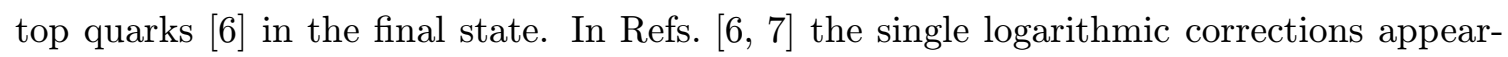
ing in the Minimal Supersymmetric Standard Model (MSSM) were included. In all these papers the gauge-invariant subset of electromagnetic corrections has been split off, and only the weak corrections have been analysed. In Ref. [푬] the weak logarithmic corrections have been separated into universal, angular-dependent, and Yukawa contributions, and the numerical importance of angular-dependent corrections was emphasized. While the total one-loop contribution remains at the level of a few per cent at $3 \mathrm{TeV}$, individual contributions reach $10 \%$. In Ref. [9.9], it was shown that the slopes of a number of observables at energies around $3 \mathrm{TeV}$ depend only on $\tan \beta$, thus potentially allowing to measure this parameter of the MSSM at CLIC for $\tan \beta<2$ and $\tan \beta>20$ with acceptable precision.

Layssac et al. [1] $\left.{ }_{1}^{1} \overline{0}\right]$ have evaluated the complete logarithmic corrections for the process $\gamma \gamma \rightarrow f \bar{f}$ including the case of heavy-quark production in the Standard Model and the MSSM. Also in this case the gauge-invariant QED corrections have been split off and the impact of the separate contributions to the weak corrections have been studied, yielding results similar to those for $\mathrm{e}^{+} \mathrm{e}^{-} \rightarrow f \bar{f}$.

\section{General result for one-loop electroweak logarithms}

A different method for the evaluation of the one-loop logarithmic corrections has been de-

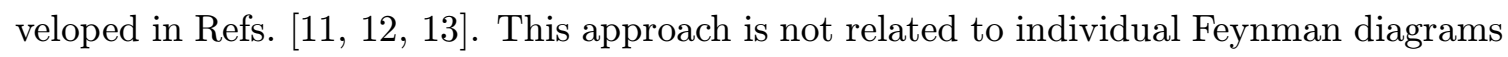
but makes use of the universality of the logarithmic corrections.

The derivation is carried out in the full spontaneously broken electroweak Standard Model. It uses the Feynman gauge and dimensional regularization with the regularization scale $\mu$ chosen as $\mu^{2}=s$. In this setup the logarithms related to the running of the couplings are obtained from the corresponding counter terms. The double-logarithmic contributions originate from those one-loop diagrams where soft-collinear gauge bosons are exchanged between pairs of external legs. These are evaluated using the eikonal approximation. The 
single-logarithmic corrections not related to parameter renormalization originate from the wave-function renormalization of the external particles and from diagrams with collinear gauge bosons attached to external lines. The latter are extracted in the collinear limit by means of Ward identities and are found to factorize the Born amplitude.

The method is applicable to non-mass-suppressed exclusive processes with arbitrary external states, including transverse and longitudinal gauge bosons as well as Higgs fields. Above the electroweak scale, the photon, Z-boson and W-boson loops are treated in a symmetric way, rather than split into electromagnetic and weak parts. To this end, the logarithms originating from the electromagnetic loops are split Ted into two parts: the contributions of a fictitious heavy photon with mass $M_{\mathrm{W}}$ are added to the W-boson and Z-boson loops resulting in the "symmetric electroweak" contribution, and the remaining part originating from the difference between the photon mass and the mass of the W-boson is denoted as "pure electromagnetic" contribution.

The results can be summarized as follows: the universal, angular-independent part of the double logarithms and the single logarithms originating from collinear diagrams and wave-function renormalization factorize the lowest-order matrix elements. The mixing of photon and $\mathrm{Z}$ boson entails, however, a mixing of the corresponding matrix elements. At $1 \mathrm{TeV}$, the corresponding relative corrections to cross sections range between $-0.1 \%$ and $-10 \%$ per external particle, the largest effects resulting from Higgs and gauge bosons. The angular-dependent logarithms, which originate from the soft-collinear region, can be associated to pairs of external lines. They do not obey a simple factorization, but require in addition matrix elements where the external particles of the original process are replaced by the corresponding SU(2) partners. Consequently, the numerical size of these corrections depends strongly on the involved matrix elements. External longitudinal gauge bosons have to be replaced by the corresponding would-be Goldstone bosons.

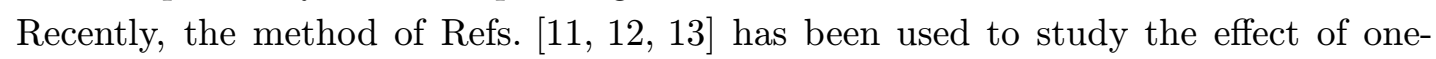
loop electroweak corrections on $\mathrm{WZ}$ and $\mathrm{W} \gamma$ production processes at the LHC [1] was found that in the physically interesting region of large transverse momentum and small rapidity separation of the gauge bosons the corrections can be calculated in the leading-pole approximation. As a result, the electroweak corrections lower the theoretical predictions by $5-20 \%$

\section{Explicit two-loop calculations}

To date, explicit two-loop calculations have essentially been performed in order to check the reliability of the resummation techniques and are restricted to the leading two-loop terms $\left[\sim \alpha^{2} \log ^{4}\left(s / M_{\mathrm{W}}^{2}\right)\right]$.

The corrections to the decay of an $\mathrm{SU}(2) \times \mathrm{U}(1)$ singlet into massless fermions have been calculated for the right-handed (abelian) case by Melles [i1 $\left.\overline{1}_{1}\right]$ and for the left-handed (non-abelian) case by Hori et al. [i $[16]$ ].

Beenakker and Werthenbach have developed a Coulomb gauge fixing for massive gauge bosons [i] $[\overline{1}]$, that permits to isolate the leading logarithms into self-energy diagrams. Explicit two-loop results for the process $\mathrm{e}^{+} \mathrm{e}^{-} \rightarrow f \bar{f}$ have been given in Ref. [1] $\left.{ }_{1}^{1} \overline{\delta_{1}}\right]$. Recently, the 
calculation of the leading two-loop logarithms for arbitrary external particles, i.e. fermions, longitudinal gauge bosons, Higgs bosons, and transverse gauge bosons has been completed

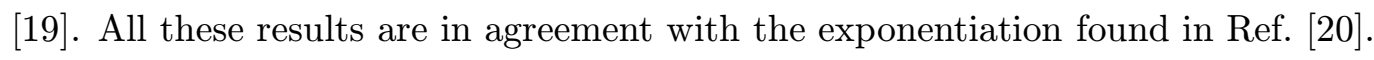

\section{Resummation of higher-order contributions}

The numerical size of the double logarithms at one loop suggests that the corresponding leading two-loop corrections can be at the level of $10 \%$ at $1 \mathrm{TeV}$. This calls for a resummation of these corrections. This resummation has mainly been studied by applying QCD resummation techniques to the electroweak theory, thereby assuming that at high energies the symmetric phase of the electroweak theory can be used. Leading $\left[\sim \alpha^{n} \log ^{2 n}\left(s / M_{\mathrm{W}}\right)\right]$, subleading $\left[\sim \alpha^{n} \log ^{2 n-1}\left(s / M_{\mathrm{W}}\right)\right]$, and recently also sub-sub-leading $\left[\sim \alpha^{n} \log ^{2 n-2}\left(s / M_{\mathrm{W}}\right)\right]$ contributions have been studied.

Fadin et al. $[\overline{2} \overline{0} \bar{j}$ have resummed the leading contributions by means of the infrared evolution equation for non-radiative processes and for processes including soft gauge-boson emission. In all cases the Sudakov double logarithms were found to exponentiate. A resum-

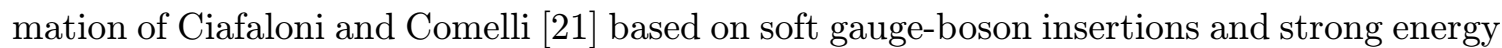
ordering gave different results. The explicit calculations mentioned in Sect. '4i-1 support the

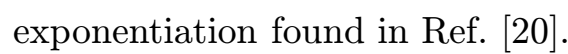

Kühn et al. have considered the process $\mathrm{e}^{+} \mathrm{e}^{-} \rightarrow f \bar{f}$ in the limit of massless fermions and have used evolution equations to resum all logarithmic corrections including the angular-dependent ones. The leading and sub-leading contributions have been evaluated in Ref. [22]. Recently also the sub-sub-leading contributions (together with the constant

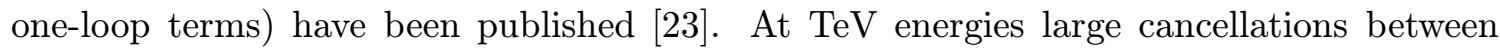
leading, sub-leading and sub-sub-leading two-loop contributions have been observed, while each of these contributions can reach several per cent at $1 \mathrm{TeV}$.

Using the concept of splitting functions, Melles has extended the infrared-evolution equation approach to the sub-leading level considering processes involving fermions and

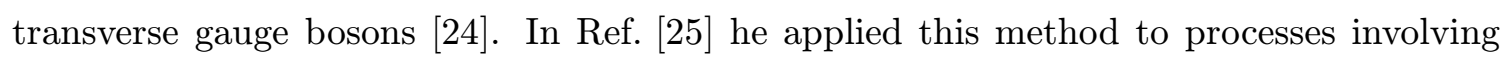
external longitudinal gauge bosons and Higgs bosons, where additional logarithms related to Yukawa couplings appear. The effect of the running gauge couplings at the sub-leading level has been discussed in Ref. [2 $2 \overline{6}]$. A review of this work can be found in Ref. [2] $\overline{7} \overline{7}]$, where it is shown that the sub-leading two-loop logarithms can yield corrections of up to $4 \%$ at $1 \mathrm{TeV}$. Very recently a generalization including also the angular-dependent logarithms for arbitrary processes has been proposed [2 $2 \overline{2}]$.

\section{Non-cancellation of leading electroweak logarithms in inclusive quanti- ties}

As discussed above, virtual one-loop corrections contain double logarithms which arise from the soft-collinear limit. In QED and QCD, these double logarithms cancel when adding the corresponding real corrections and, in case of QCD, averaging over the colours of the initial state particles, such that inclusive quantities are free of double logarithms. It has been 
pointed out in Ref. [2] $\bar{g}]$, that the situation is different in the electroweak Standard Model. Because the initial states $\left(\mathrm{e}^{+} \mathrm{e}^{-}, \mathrm{qq}^{\prime}, \gamma \gamma, \ldots\right)$ carry a definite non-abelian flavour, the double logarithms do not cancel in inclusive hard cross sections and the Bloch-Nordsieck theorem is violated.

The origin of this non-cancellation can be understood by considering, for instance, fully inclusive $\mathrm{e}^{+} \mathrm{e}^{-}$annihilation: virtual corrections simply multiply the lowest-order matrix elements and are proportional to the lowest-order cross section $\sigma_{\mathrm{e}^{+} \mathrm{e}^{-}}$. The same holds for the corrections related to real emission of a photon or a $\mathrm{Z}$ boson. However, the real emission of a charged $\mathrm{W}$ boson changes the isospin of the incoming electron and turns it into a neutrino. The corresponding double-logarithmic corrections are proportional to the cross section $\sigma_{\mathrm{e}^{+} \nu_{\mathrm{e}}}$ and do not cancel the corresponding virtual corrections. In fact, the resulting electroweak corrections exceed the QCD corrections in the $\mathrm{TeV}$ range.

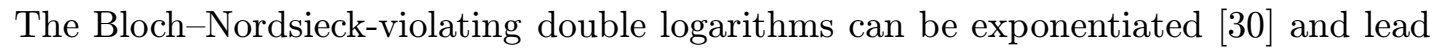
to suppression of cross-section differences within weak-isospin multiplets. Recently, it has been found that Bloch-Nordsieck violation can also occur in spontaneously broken abelian gauge theories, if the incoming particles are mass eigenstates that do not coincide with gauge eigenstates [i] $\left[\begin{array}{l}3 \\ 1\end{array}\right]$. In the Standard Model this mechanism becomes relevant for incoming longitudinal gauge bosons or Higgs bosons [32] $\left.{ }^{3}\right]$, since these can turn into each other via the emission of a $\mathrm{Z}$ boson.

\section{Conclusions}

At energies above the electroweak scale electroweak logarithms can lead to large corrections of several tens of per cent. Unlike in QED and QCD, the leading double-logarithmic corrections do not cancel in inclusive quantities. On the other hand, at $\mathrm{TeV}$ energies leading, sub-leading, and sub-sub-leading virtual corrections tend to cancel each other, and the size of the total corrections depends strongly on the energy and on the observable under consideration. It is clear that these corrections must be under control in order to analyse experiments at future colliders.

The virtual electroweak logarithmic corrections at one loop have been calculated for various processes, and a general method for the evaluation of these corrections has been developed. The leading double-logarithmic corrections can be resummed via exponentiation of the one-loop expression. First results for the sub-leading and sub-sub-leading corrections at two loops exist. The investigation of these corrections requires further studies.

\section{Acknowledgments}

I would like to thank S. Pozzorini for help in the preparation of the manuscript.

\section{References}

[1] M. Kuroda, G. Moultaka and D. Schildknecht, iNucl. $\bar{P} \bar{h} \bar{s}$. B $3 \overline{5} \overline{0}(1 \overline{9} \overline{1} \overline{1}) \overline{2}$;

G. Degrassi and A. Sirlin, 'Phys. Rev. D $46-1992) 3104$

A. Denner, S. Dittmaier and R. Schuster, Nucl. Phys. B $\mathbf{4} 52$ - 19955$) 80$; 
A. Denner, S. Dittmaier and T. Hahn, Phys. Rev. D 56 6 1997$) 117$;

A. Denner and T. Hahn, №cl. Phys. B $\mathbf{5 2 5}(1998) 2 \pi$.

[2] W. Beenakker et al., №cl. Phys. B 410_(1993) 245i 'Phys. Lett. B 317. (1993) 622.

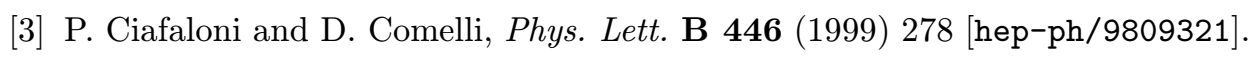

[4] M. Beccaria et al., Phys. Rev. D'61 (2000) 073005, [hep-ph/9906319].

[5] M. Beccaria et al. Phys. Rev. D'61 (2000) 011301! hep-ph/9907389].

[6] M. Beccaria, F. M. Renard and C. Verzegnassi, Phys. Rev D $63-(2001) 053013$ [hep-ph/0010205is.

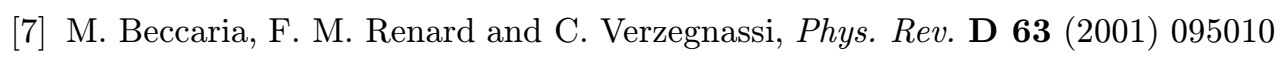
hep-ph/0007224].

[8] M. Beccaria, F. M. Renard and C. Verzegnassi, hep-ph/0103335i.

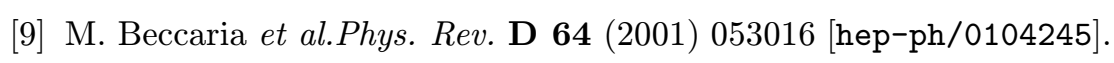

[10] J. Layssac and F. M. Renard, Phys. Rev. D 64 4 (2001) 053018i hep-ph/0104205].

[11] A. Denner and S. Pozzorini, 'Eur. Phys. J. 18 .

[12] A. Denner and S. Pozzorini, Eur. Phys. J. C 21 (2001) 633 [hep-ph/0104127].

[13] S. Pozzorini, dissertation, Universität Zürich, 2001.

[14] E. Accomando, A. Denner and S. Pozzorini, hep-ph/0110114t

[15] M. Melles, 'Phys. Lett. B 495 $2000[81$ i hep-ph/0006077].

[16] M. Hori, H. Kawamura and J. Kodaira, Phys. Lett. B 491 $(2000) 2751$ [hep-ph/0007329].

[17] W. Beenakker and A. Werthenbach, Nucl. Phys. Proc. Suppl. 89 (2000) 88 [hep-ph/0006009].

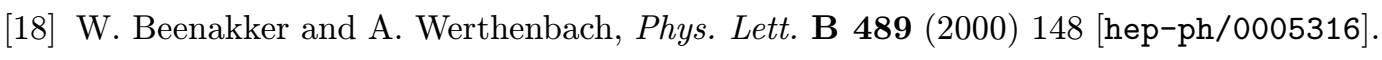

[19] W. Beenakker and A. Werthenbach, DESY 01-131.

[20] V. S. Fadin et al., 'P

[21] P. Ciafaloni and D. Comelli, 'Phys. Lett. B 4766 (2000) $49 \overline{9}$, linep-ph/9910278].

[22] J. H. Kühn, A. A. Penin and V. A. Smirnov, 'Eur. Phys. J. C 17.(2000) 971 hep-ph/9912503].

[23] J. H. Kühn et al., hep-ph/0106298:

[24] M. Melles, iPhys. Rev. D-63 (2001) 034003i [hep-ph/0004056];

[25] M. Melles, iPhys. Rev. D 64 4 $(2001) 014011$, hep-ph/0012157].

[26] M. Melles, Physs. Rev. D 64 (2001) 054003 [hep-ph/0102097i].

[27] M. Melles, hep-ph/0104232i.

[28] M. Melles, hep-ph/0108221!

[29] M. Ciafaloni, P. Ciafaloni and D. Comelli, 'Phys. Rev. Lett. 84 (2000) 4810' [hep-ph/0001142].

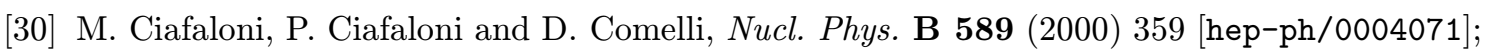

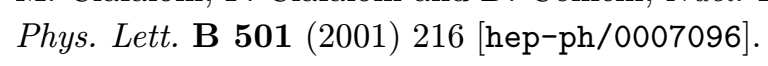

[31] M. Ciafaloni, P. Ciafaloni and D. Comelli, hep-ph/0103315i.

[32] M. Ciafaloni, P. Ciafaloni and D. Comelli, iNucl. Phys. B 613(2001) 382i hep-ph/0103316i. 